\title{
A NOTE ON RINGS WITH CERTAIN VARIABLE IDENTITIES
}

\section{HAZAR ABU-KHUZAM}

Department of Mathematical Sciences

King Fahd University of Petroleum \& Minerals Dhahran, Saud 1 Arabia

(Paper received on June 30, 1987 and in revised form on October 29, 1988)

ABSTRACT. It is proved that certain rings satisfying generalized-commutator constraints of the form $\left[x^{m}, y^{n}, y^{n}, \ldots, y^{n}\right]=0$ with $m$ and $n$ depending on $x$ and $y$, must have nil commutator ideal.

KEY WORDS AND PHRASES. Commutator ideal, periodic ring. 1980 AMS SUBJECT CLASSIFICATION CODE. $16 \mathrm{~A} 70$.

\section{INTRODUCTION.}

Let $\left[x_{1}, x_{2}\right]$ denote $x_{1} x_{2}-x_{2} x_{1}$, and for $k>2$, let $\left[x_{1}, x_{2}, \ldots, x_{k}\right]=$ $\left[\left[x_{1}, \ldots, x_{k-1}\right], x_{k}\right]$. For $x_{1}=x$ and $x_{2}=x_{3}=\ldots=x_{k}=y$, denote $[x, y, \ldots, y]$ by $[x, y]_{k} \cdot A$ result of Herstein [1] and of Anan'in and Zyabko [2] asserts that if for any $x$ and $y$ in a ring $R$, there exist positive integers $m=m(x, y)$, $n=n(x, y)$ such that $x^{m} y^{n}=y^{n} x^{m}$, then the commutator ideal of $R$ is $n i l$. Recently, Herstein [3] proved that a ring $R$ in which for any $x, y, z \in R$ there exists positive integers $m=m(x, y, z), n=n(x, y, z)$, and $q=q(x, y, z)$ such that

$\left[\left[x^{m}, y^{n}\right], z^{q}\right]=0$ must have nil commutator ideal. More recently klein, Nada and Bel1 [4] raised the following conjecture which arises naturally from the above mentioned work.

CONJECTURE. Let $k>1$. If for each $x, y \in R$, there exists positive integers $m$ and $n$ such that $\left[x^{m}, y^{n}\right]_{k}=0$, then the commutator ideal of $R$ is $n 11$.

In [4], Klein, $\mathrm{Nada}$ and Bell proved the conjecture for rings with identity 1. Given the complexity of [1] and [3], it would appear that no proof of this conjecture is in sight. Our objective is to prove the conjecture for certain classes of rings and to generalize a result of Herstein in [3] and some results in [4] and [5].

A ring $R$ is called periodic if for each $x$ in $R$, there exists distinct positive integers $m$ and $n$ for which $x^{m}=x^{n}$. In preparation for the proofs of our main theorems, we start with the following lemma which is known [5] and we omit its proof. 
LEMMA 1. If $R$ is a periodic ring, then for each $x$ in $R$, there exists a positive integer $k=k(x)$ such that $x^{k}$ is idempotent.

\section{MAIN RESULTS.}

The following theorem shows that the conjecture is true for Artinian rings.

THEOREM 1. Let $k>1$, and let $R$ be an Artinian ring such that for each $x, y$ in $R$, there exists positive integers $m$ and $n$ such that $[x, y]_{k}=0$. Then the commutator ideal of $R$ is $n i l$.

PROOF. To prove that the commutator ideal of $R$ is $n i l$ it is enough to show that if $R$ has no nonzero $n i l$ ideals then it is commutative. So we suppose that $R$ has no nonzero nil ideals. Since $R$ is Artinian, the Jacobson radical $J$ of $R$ is nilpotent. So $J=0$, and hence $R$ is semisimple Artinian. This implies that $R$ has an identity element and now, $R$ is commutative by Theorem 3 of [4].

Next, we prove Theorem 2 which shows that the conjecture is true for periodic rings. This result generalizes a result of Bell in [5].

THEOREM 2. Let $k>1$ and 1 et $R$ be a periodic ring such that for each $x, y$ in $R$ there exists positive integers $m$ and $n$ such that $\left[x^{m}, y^{n}\right]_{k}=0$. Then the commutator ideal of $R$ is nil.

PROOF. If $k=2$, then the result follows by the theorem in [1]. So assume $k>2$ and let $x$ be any element of $R$ and let $e$ be any idempotent of $R$. By hypothesis, there exists integers $m$ and $n$ such that $\left[x^{m}, e^{n}\right]_{k}=0$. This implies that $\left[x^{m}, e\right]_{k}=0$, and hence

$$
\left[x^{m}, e\right]_{k-1} e=e\left[x^{m}, e\right]_{k-1}
$$

Multiplying by $e$ from the right and using the fact that $e\left[x^{m}, e\right]_{k-1} e=0$ we obtain $\left[x^{m}, e\right]_{k-1} e=0$. Hence $0=\left(\left[x^{m}, e\right]_{k-2} e-e\left[x^{m}, e\right]_{k-2}\right) e^{-1}=\left[x^{m}, e\right]_{k-2} e$. Continuing this way we get $\left[x^{m}, e\right] e=0$ which implies that $x^{m} e=e^{m}$ e. Similarily, we can get $\operatorname{ex}^{m}=\operatorname{ex}^{m}$ e. This implies that

$$
x^{m} e=e x^{m}, x \in R \text {, e any Idempotent and } m=m(x, e) \text {. }
$$

Let $y$ be any element of $R$. Since $R$ is periodic, Lemma 1 implies that $y^{p}$ is idempotent for some positive integer $p=p(y)$. So (2.1) implies that for each $x, y$ in $R$ there exists positive integers $m$ and $p$ such that $x^{m} y^{p}=y^{p} x^{m}$. Now, the result follows by the we11-known theorem in [1] or [2].

THEOREM 3. Let $k>1$. If $R$ is a prime ring having a nonzero idempotent element such that for each $x, y$ in $R$ there exists positive integers $m$ and $n$ such that $\left[x^{m}, y^{n}\right]_{k}=0$. Then $R$ is commutative.

PROOF. The argument used in Theorem 2 to reach statement (2.1) in the proof shows that a ring satisfying the generalized commutator constraint $\left[x^{m}, y^{n}\right]_{k}=0$ must have its idempotent elements in the center. For let $e_{1}$ and $e_{2}$ be idempotent elements in R. (2.1) implies that $e_{1} e_{2}=e_{2} e_{1}$ and hence the idempotents of $R$ commute. This implies that the idempotents of $R$ are central in $R$ [6, Remark 2]. Let e be a nonzero 
idempotent of $R$. Then $e$ is a nonzero central idempotent in the prime ring $R$. Hence $e$ is an identity element of $R$ since it can not be a zero divisor. Now $R$ is commutative by Theorem 3 of [4].

The proof of Theorem 4 below was done by Kezlan in the proof of his main theorem in [7]. So we omit its proof here.

THEOREM 4. Let $k>1$. If $R$ is a prime ring with a nontrivial center such that for each $x, y$ in $R$ there exists positive integers $m$ and $n$ such that $\left[x^{m}, y^{n}\right]_{k}=0$, then $R$ is commutative.

The following result generalizes Theorem 1 of [4].

THEOREM 5. Let $R$ be a ring and let $M$ be a fixed positive integer. Suppose that for each $x, y \in R$ there exist positive integers $m=m(x, y)<M$ and $n=n(x, y)$ such that $\left[x^{m}, y^{n}, y^{n}\right]$ belongs to the center of $R$. Then the commutator ideal of $R$ is $n i l$.

PROOF. Again, we suppose that $R$ has no $n i l$ ideals and hence $R$ is a subdirect product of prime rings satisfying the above hypothesis of $R$. So we may assume that $R$ is prime. Let $Z$ be the center of $R$. If $Z=0$, then for each $x, y \in R$, $\left[x^{m}, y^{n}, y^{n}\right]=0$ where $m=m(x, y)<M$, and $n=n(x, y)$. This implies that $R$ is commutative by Theorem 1 of [4]. So we may assume that $R$ has a nontrivial center, and hence $R$ is commutative by Theorem 4 above.

The following result generalizes Theorem 8 in [3].

THEOREM 6. Let $R$ be a ring in which, for each $x, y, z \in R$, there exists positive integers $m=m(x, y, z) n=n(x, y, z)$ and $q=q(x, y, z)$ such that $\left[x^{m}, y^{n}, z^{q}\right]$ belongs to the center of $R$. Then the commutator ideal of $R$ is $n i l$.

PROOF. Again, we may assume that $R$ is a prime ring satisfying the above hypothesis. Let $\mathrm{Z}$ be the center of $\mathrm{R}$. If $\mathrm{Z}=0$, then for each $x, y, z \in R$, $\left[\left[x^{m}, y^{n}\right], z^{q}\right]=0$, where $m=m(x, y, z), n=n(x, y, z)$ and $q=q(x, y, z)$. This implies that $R$ is commutive by Theorem 8 of [3]. So we may assume that $R$ has a nontrivial center. For any $x, y$ in $R,\left[\left[x^{m}, y^{n}\right], y^{q}\right] \in z$ where $m, n$, are each functions of the variables $x$ and $y$. So $\left.\left[\left[x^{m}, y^{n}\right], y^{q}\right], y\right]=0$, which implies that $\left.\left[\left[x^{m}, y^{n q}\right], y^{n q}\right], y^{n q}\right]=0$. Hence $R$ is commutative by Theorem 4 above.

REMARK. The result in Theorem $6 \mathrm{c}$ an be generalized as follows. Let $R$ be a ring such that for each $x, y, z \in R$, there exists positive integers $m=m(x, y, z), n=$ $n(x, y, z)$ and $q=q(x, y, z)$ such that $\left[x^{m}, y^{n}, z^{q}, r_{1}, r_{2}, \ldots, r_{k}\right]=0$ for all elements $r_{1}, \ldots, r_{k}$ in $R$. Then the commutator ideal of $R$ is $n i 1$. This can be done by induction on $k$ and using the argument in Theorem 6 . We omit the detalls of the proof.

ACKNOWLEDGEMENT. This research is supported by King Fahd University of Petroleum and Minerals. 


\section{REFERENCES}

1. HERSTEIN, I.N., A Commutativity Theorem, J. Algebra, 38(1976), 112-118.

2. ANAN'IN, A.Z. \& ZYABKO, E.M., On a Question of Faith, Algebra 1 Logica $13(1974), 125-131$.

3. HERSTEIN, I.N., On Rings with a Particular Variable Identity, J. Algebra $62(1980), 346-357$.

4. KLEIN, A., NADA, I. \& BELL, H., Some Commutativity Results for Rings, Bull. Austra 1. Math. Soc., 22(1980), 285-289.

5. BELl, H.E., Some Commutativity Results for Periodic Rings, Acta Math. Acad. Sc1. Hungar., 28(1976), 279-283.

6. HIRANO, Y., TOMINAGA, H. \& YAQUB, A., On Rings Satisfying the Identity $\left(x+x^{2} \ldots+x^{n}\right)^{(n)}=0$, Math. J. Okayama Univ. $25(1983), 13-18$.

7. KEZLAN, T.P., On Generalized Commutators and Nil Commutator Ideals, Arch. Math. 43(1984), 21-24.

8. HERSTEIN, I.N., Rings with Involution, University of Chicago Press, Chicago, 1976. 


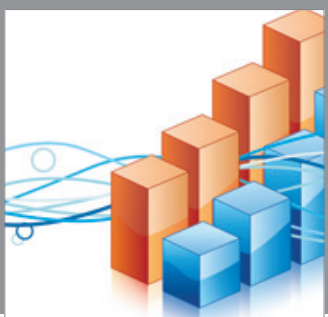

Advances in

Operations Research

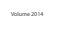

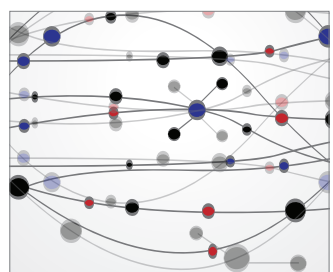

\section{The Scientific} World Journal
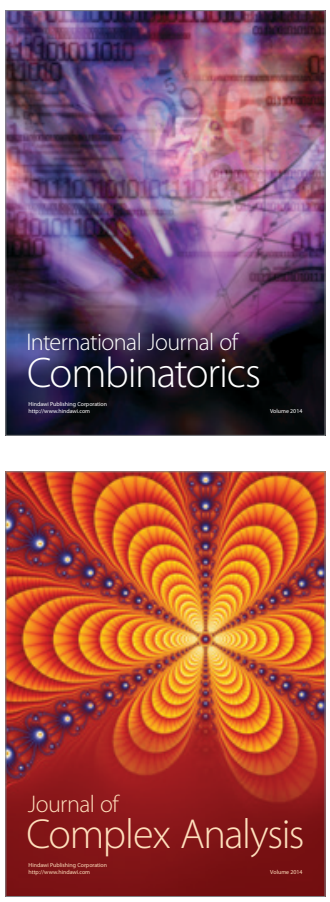

International Journal of

Mathematics and

Mathematical

Sciences
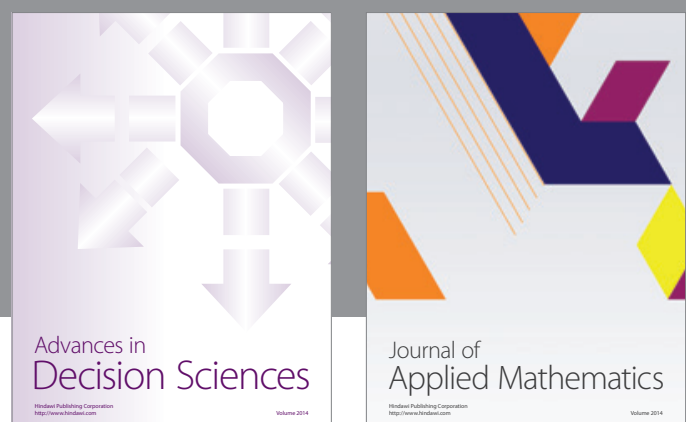

Journal of

Applied Mathematics
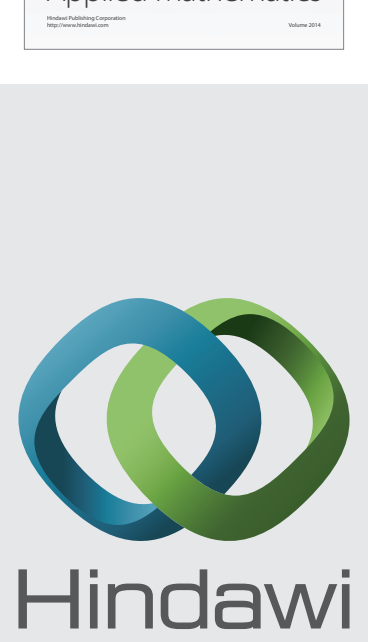

Submit your manuscripts at http://www.hindawi.com
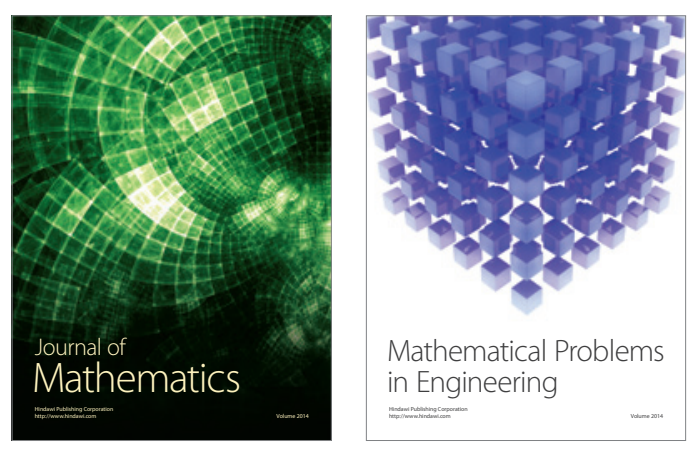

Mathematical Problems in Engineering
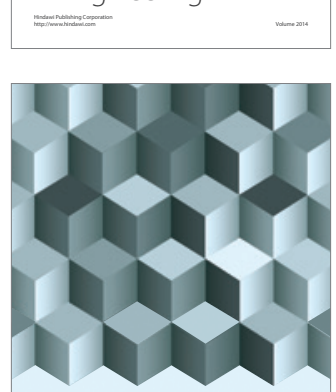

Journal of

Function Spaces
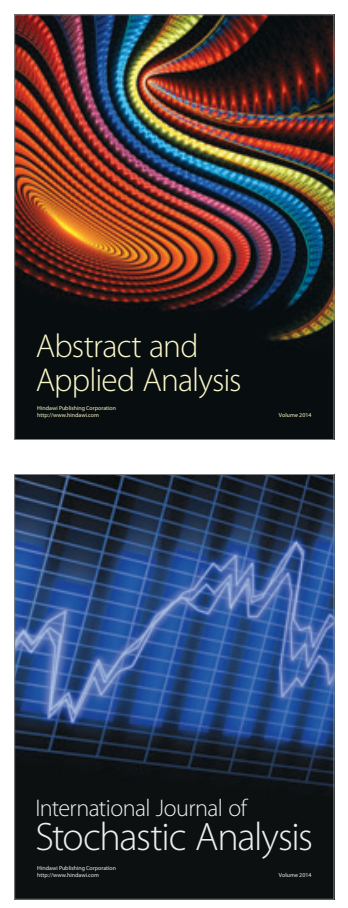

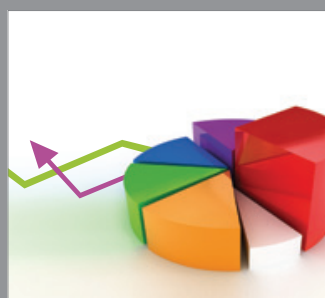

ournal of

Probability and Statistics

Promensencen
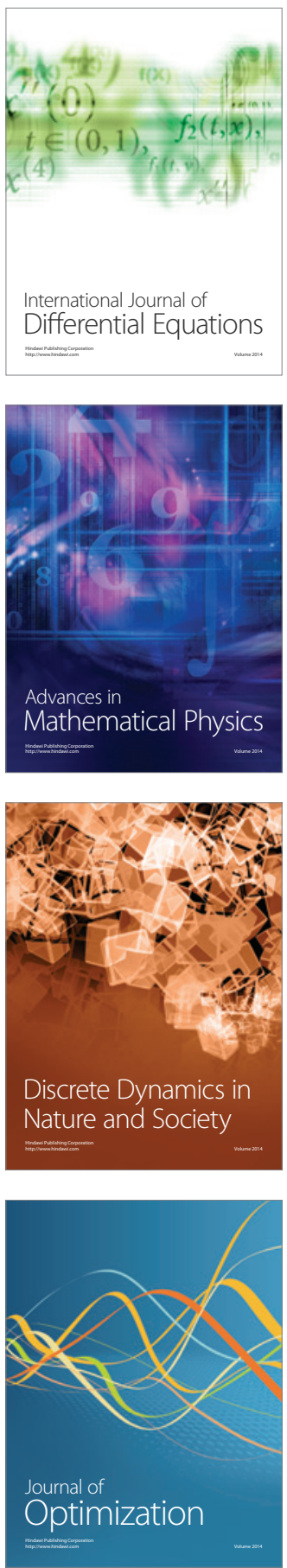